\title{
Relação entre bromélias epifíticas e forófitos em diferentes estádios sucessionais
}

Relationship between epiphytic bromeliads and phorophytes at different successional stages

\author{
Marcia Patricia Hoeltgebaum ${ }^{1,2,3}$, Maike Hering de Queiroz ${ }^{\dagger} \&$ Maurício Sedrez dos Reis ${ }^{2}$
}

\begin{abstract}
Resumo
O presente estudo teve como objetivo analisar a relação entre as bromélias epifíticas e a vegetação arbustiva e arbórea registrada em diferentes estádios sucessionais da Floresta Ombrófila Densa, com o intuito de detectar forófitos potenciais à colonização por bromélias. Os forófitos foram selecionados por meio do método de quadrantes centrados, e verificada a relação entre as suas características morfológicas com a ocorrência de bromélias, por meio da análise de Correlação de Spearman e teste de qui-quadrado. Foram registradas 27 espécies bromélias epifíticas pertencentes a oito gêneros, e 85 espécies forofíticas. $\mathrm{O}$ diâmetro foi a variável que apresentou maior influência na riqueza das bromélias. Forófitos com cascas persistentes e com textura rugosa apresentaram maior frequência de bromélias. Palavras-chaves: árvore hospedeira, bromeliaceae, epífitas, Floresta Ombrófila Densa.
\end{abstract}

\begin{abstract}
The main objective of this study was to identify the relationship between epiphytic bromeliad species and their associated native forest vegetation (trees and shrubs) at different successional stages in the southern coastal Atlantic Rainforest. This study also characterized potential colonization of phorophytes in identified epiphytic bromeliads species. The phorophytes were selected by the phytosociological sampling point-centered quarter method. The relationship between the phorophytes morphological characteristics and epiphytic bromeliads species occurrence was analyzed using a Spearman Correlation and chi-square tests. Twenty-seven epiphytic bromeliads species representing eight genera were found on eighty-five phorophytes. The variable phorophyte diameter presented the greatest influence on the abundance of epiphytic bromeliads species found. Phorophytes with a persistent bark and rough texture showed higher frequency of bromeliads.
\end{abstract}

Key words: host tree, bromeliaceae, epiphytes, Atlantic Rainforest.

\section{Introdução}

A família Bromeliaceae destaca-se por sua ocorrência em uma extensiva diversidade de hábitats e por sua capacidade em ocupar ambientes com baixa disponibilidade de água e nutrientes (Pittendrigh 1948). As adaptações morfológicas, fisiológicas e anatômicas presentes nesta família, possibilitaram sua independência do solo, contribuindo desta forma, para o pronunciado epifitismo observado entre suas espécies (Benzing \& Renfrow 1974; Akinsoji 1990).

As bromélias constituem um dos componentes mais marcantes das florestas tropicais americanas, contribuindo significativamente para a manutenção da diversidade biológica e o equilíbrio interativo entre as espécies (Benzing 2000). A riqueza e abundância dessas espécies em um determinado lugar podem ser utilizadas para estimar o status de conservação do ambiente (Leme \& Marigo 1993), uma vez que respondem com diferenças na composição e abundância de espécies em diferentes formações florestais e também sobre os forófitos que as suportam (Ter Steege \& Cornelissen 1989; Hietz 1997).

A importância e a contribuição dos forófitos para a riqueza e a distribuição espacial de epífitas têm sido relatadas em estudos que buscam elucidar fatores ecológicos e processos que conduzem a padrões de diversidade observados no componente epifitico (Hirata et al. 2009). Diversos autores demonstram que epífitas diferem em abundância entre forófitos (Benzing 1981; Bennett 1986; Ter Steege \& Cornelissen 1989; Migenis

\footnotetext{
${ }^{1}$ Parte da dissertação de Mestrado desenvolvida na Universidade Federal de Santa Catarina, Programa de Pós-Graduação em Biologia Vegetal.

${ }^{2}$ Universidade Federal de Santa Catarina, Centro de Ciências Agrárias, Depto. Fitotecnia, Núcleo de Pesquisas em Florestas Tropicais - NPFT, Rod. Admar Gonzaga 1346, Itacorubi, 88034-001, Florianópolis, SC, Brasil.

${ }^{3}$ Autor para correspondência: patibio@ig.com.br
} 
\& Ackerman 1993; Dejean et al. 1995; Kernan \& Fowler, 1995; Fontoura et. al 2009). Segundo Benzing (1995a), estudos localizados eventualmente podem demonstrar preferências de epífitos por forófitos. Tal comportamento foi encontrado em alguns trabalhos (Zimmerman \& Olmsted 1992; Hietz-Seifert et al. 1996; Callaway et al. 2002). Contudo, os resultados obtidos na maioria dos estudos demonstram que a presença e abundância de epífitas estão associadas a características das espécies hospedeiras (Frei \& Dodson 1972; Schlesinger \& Marks 1977; Medeiros \& Jardim 2011), como arquitetura, textura e estabilidade da casca (Gentry \& Dodson 1987; Hietz \& Hietz-Seifert 1995; Fontoura et al. 1997; Nieder et al. 2000; Kersten et al. 2009), e também pelo tempo em que eles estão disponíveis à colonização no ambiente (Benzing 1995a; Bonnet et al. 2007). Hirata et al. (2009) sugerem que o tamanho, estabilidade, e qualidade do hospedeiro são igualmente importantes na determinação da colonização epífitas. Entretanto, quando se considera a família Bromeliaceae, pouco se analisou sobre possíveis especificidades e relações com os forófitos e suas características.

O conhecimento da diversidade das bromélias epifíticas em diferentes estádios da sucessão da floresta e um melhor entendimento das condições forofíticas específicas para a sua colonização, podem contribuir para o aprimoramento dos trabalhos de restauração ambiental, nos quais seu uso ainda não é empregado. Assim, o presente estudo teve como objetivo analisar a relação entre as bromélias epifíticas e a vegetação arbustiva e arbórea registrada em diferentes estádios da sucessão da floresta, com o intuito de detectar forófitos potenciais à colonização por bromélias.

\section{Material e Métodos}

Área de estudo

O presente estudo foi desenvolvido no ano de 2002, no Parque Botânico do Morro Baú, localizado nos municípios de Ilhota e Luiz Alves (26 $47^{\prime} 10^{\prime \prime}$ e $26^{\circ} 50^{\prime} 15^{\prime \prime}$ 'S e $48^{\circ} 55^{\prime} 33^{\prime \prime}$ e 48 $57^{\prime} 25^{\prime \prime} \mathrm{W}$ ), estado de Santa Catarina. O clima da região é tropical úmido, com uma temperatura média anual de $20^{\circ} \mathrm{C}$ (Gaplan 1986). A precipitação média anual está entre $1.800 \mathrm{e}$ $2.000 \mathrm{~mm}$ com chuvas bem distribuídas ao longo do ano, sem período de seca e com maior intensidade no verão. A umidade relativa do ar é muito elevada, com valores entre 84 a $86 \%$ conseqüência dos frequentes nevoeiros que mantém a parte superior do Morro Baú encoberta (Klein 1980). A região do Parque encontra-se sobre a Formação Baú, que pertence ao Grupo Itajaí do Complexo Luiz Alves, parte do Escudo Catarinense (Gaplan 1986; Possamai 1989). O solo é do tipo Cambissolo Álico, não hidromórfico, de baixa fertilidade e de textura argilosa, com relevo ondulado e montanhoso (Gaplan 1986).

Aárea constitui um mosaico de formações em diversos estádios sucessionais da Floresta Ombrófila Densa montana e alto montana (Klein 1980), resultado de cortes seletivos e rasos efetuados em diferentes momentos da história da área. A delimitação dos diferentes estádios sucessionais e Floresta Primária seguiu os critérios propostos por Klein (1980). Foram delimitados em: a) Capoeirinha - área em $268 \mathrm{~m}$ de altitude, caracteriza-se pela dominância de plantas arbustivas como Baccharis dracunculifolia DC (Asteraceae), Tibouchina pilosa Cogn. e T. urvilleana (DC) Cogn. (Melastomataceae); encontram-se ainda plantas herbáceas e indivíduos jovens de espécies arbóreas de estádios mais avançados como Myrsine coriacea Nad. e Miconia cinnamomifolia Cambess. A área foi anteriormente utilizada para o plantio de aipim e cana por cerca de 10 anos consecutivos e estava abandonada há dois anos. Segundo a Resolução n ${ }^{\circ}$ 04/94 do CONAMA equivale ao estágio inicial de regeneração; b) Capoeira - área em $281 \mathrm{~m}$ de altitude, vegetação com componente herbáceo menos expressivo e domínio marcante de Myrsine coriacea (Myrsinaceae) acompanhada por Clusia criuva (Clusiaceae). A regeneração da floresta nesta área teve início há 10 anos e equivale ao estágio médio de regeneração; c) Capoeirão - área em 275 $\mathrm{m}$ de altitude, com vegetação caracterizada pela instalação e domínio de Miconia cinnamomifolia (DC.) Naud. (Melastomataceae), acompanhada por M. cabucu W. Hoehne (Melastomataceae), Alchornea triplinervia Muell. Arg. (Euphorbiaceae), entre outras. A área estudada possui cerca de 20 anos de idade. É denominado pelo CONAMA como estágio avançado de regeneração; d) Floresta Secundária - área em $338 \mathrm{~m}$ de altitude, onde destacam-se as espécies: Tapirira guianensis Aubl. (Anacardiaceae), Cabralea canjerana (Vell.) Mart. (Meliaceae), Hieronyma alchorneoides Fr. All. (Euphorbiaceae), Sloanea guianensis Benth. (Elaeocarpaceae) e, no estrato médio podem ser encontrados vários indivíduos de Euterpe edulis Martius entre outras espécies típicas desta fase sucessional (Klein 1980; Queiroz 1994). A área sofreu intenso corte seletivo de praticamente todas as espécies madeiráveis há cerca de 50 anos; e) Floresta Primária - área em 542 $\mathrm{m}$ de altitude, sem registro de interferência antrópica; vegetação com espécies típicas para estas formações como Ocotea catharinensis Mez (Lauraceae) e 
Sloanea guianensis Benth. (Eleoacarpaceae) com elevado DAP (diâmetro à altura do peito) e estrutura semelhante à descrita por Klein (1980).

\section{Amostragem}

Cada forófito foi considerado uma unidade amostral, definido pelo método de quadrantes centrados (Mueller-Dombois \& Ellenberg 1974; Krebs 1999). Foram amostrados 15 pontos em cada estádio, distribuídos a cada $10 \mathrm{~m}$ ao longo de transectos demarcados paralelamente a 20 metros de distância entre si, totalizando 60 forófitos por ambiente de estudo (Waechter 1992). O critério para inclusão do forófito foi o diâmetro à altura do peito (DAP) $\geq 5 \mathrm{~cm}$ para o estádio Capoeira e Capoeirão, $\geq 10 \mathrm{~cm}$ para a Floresta Secundária e $\geq 15 \mathrm{~cm}$ para a Primária. Para espécies não arbóreas do primeiro estádio foram inclusos os indivíduos com o diâmetro à altura do solo $(\mathrm{DAS}) \geq 2 \mathrm{~cm}$. Foi registrada a altura total dos forófitos amostrados e estes, caracterizados quanto à aspereza e persistência da casca, conforme classificação proposta por Waechter (1992) e Torres et al. (1994) em: lisa, áspera, rugosa e fissurada, e, ainda, em: persistente ou descamante. Em cada unidade amostral, foram registradas as espécies de bromélias presentes, bem como a presença de plântulas. Para as espécies forofíticas amostradas, foram calculadas a frequência absoluta $(\mathrm{Nai}=100(\mathrm{Nfi} / \mathrm{Nfa})$ e relativa $(\mathrm{Nri}=100$ (Nfi/åNfi)) (Mueller-Dombois \& Ellenberg 1974; Braun-Blanquet 1979; Martins 1993).

Os ramos dos forófitos e as bromélias férteis foram depositados no Herbário da Universidade Federal de Santa Catarina - (FLOR). Para a determinação das espécies, contou-se com a ajuda de especialistas e consulta em bibliografias específicas. Para registro e identificação das bromélias, foi utilizado, quando necessário, binóculo $(7 \times 35 \mathrm{~mm})$ e técnicas de arvorismo. A altura dos forófitos foi mensurada com régua dendrométrica até cerca de 12 $\mathrm{m}$ de altura e quando o forófito excedia esta medida, as mesmas foram estimadas.

As espécies epifíticas foram caracterizadas quanto ao estágio de desenvolvimento, sendo categorizadas como plântulas todas as bromélias epífitas jovens, morfologicamente diferentes da espécie adulta e que não puderam ser identificadas no nível genérico (Bonnet \& Queiroz 2006) e como adultas, as espécies identificáveis e já diferenciadas das plântulas.

\section{Análise estatística}

Para avaliar a potencialidade dos forófitos à colonização foi verificada a relação entre bromélias epifíticas (plântulas e adultas) com as características morfológicas do forófito (DAP, altura e padrão de casca). A relação entre o número de espécies epifíticas e o diâmetro, e a altura do forófito foi avaliada pela de análise de Correlação de Spearman (Sokal \& Holf 1969). Preferências entre a presença de bromélias epifíticas e o padrão de casca, foram testadas pelo qui-quadrado, utilizando tabela de contingência (Zar 1996).

\section{Resultados}

Foi registrado um total de 27 espécies de bromélias pertencentes a oito gêneros e a duas subfamílias: Tillandsioideae com 16 espécies (59\%) e Bromelioideae com 11 espécies (41\%) (Tab. 1). O gênero com o maior número de táxons foi Vriesea.

Houve diferenças na riqueza de bromélias nos diferentes estádios da regeneração florestal. A Floresta Primária apresentou o maior número de espécies (24). Na Floresta Secundária foram registradas 19 espécies e no Capoeirão, apenas 11 espécies. $\mathrm{Na}$ Capoeira foram registradas apenas plântulas enquanto que na Capoeirinha não foi constatada a presença de bromélias adultas e nem de plântulas.

A vegetação arbustiva e arbórea amostrada nas áreas estudadas apresenta diferentes características, conforme as espécies que as constituem e o estádio sucessional em que ocorrem. $\mathrm{O}$ aumento do diâmetro e da altura dos forófitos amostrados evidencia-se em direção à Floresta Primária, onde se encontram as espécies de maior porte bem como o maior número de espécies registradas (Tab. 2). Os forófitos amostrados foram representados por 85 espécies (Tab. 3).

Considerando a relação das bromélias epifíticas com parâmetros forofíticos, os valores obtidos pela análise de correlação indicam que em todas as formações com ocorrência de bromélias epifíticas, a relação entre o DAP e o número de espécies de bromélias é mais forte comparado com a altura (Tab. 4). Os valores de correlação foram maiores nos estádios mais avançados de estádio sucessional.

Com relação aos padrões de casca, a persistência, principalmente para o estabelecimento de plântulas, e a rugosidade foram os que apresentaram maior frequência de bromélias. Estes padrões são os encontrados nas espécies forofíticas mais frequentes em cada estádio (Tab. 5).

\section{Discussão}

O número de bromélias epifíticas amostradas neste estudo é bastante significativo, quando considerados outros levantamentos realizados 
Tabela 1 - Bromélias epifíticas amostradas pelo método de quadrantes centrados nos diferentes estádios sucessionais da Floresta Ombrófila Densa, Parque Botânico do Morro Baú-Ilhota/SC. (CAP1= Capoeirinha; CAP2= Capoeira; CAP 3 = Capoeirão; FS = Floresta Secundária e FP = Floresta Primária).

Table 1 - Epiphytic bromeliads sampled by the point-centered quarter method procedure at different successional stages in the Atlantic Rainforest at Morro do Baú Botanical Park (Ilhota, Santa Catarina State - Brazil).(CAP1= Capoeirinha; CAP2= Capoeira; CAP3= Capoeirão; FS = Secundary Forest e FP = Primary Forest).

\begin{tabular}{|c|c|c|c|}
\hline Espécies & CAP1 CAP2 & CAP3 & FS \\
\hline \multicolumn{4}{|l|}{ Subfamília Bromelioideae } \\
\hline \multicolumn{4}{|l|}{ Aechmea blumenavii Reitz } \\
\hline \multicolumn{4}{|l|}{ Aechmea caudata Lindman } \\
\hline \multicolumn{4}{|l|}{ Aechmea cylindrata Lindman } \\
\hline \multicolumn{4}{|c|}{ Aechmea nudicaulis (Linnaeus) Griseb. } \\
\hline \multicolumn{4}{|c|}{ Aechmea ornata (Gaudichaud) Baker } \\
\hline \multicolumn{4}{|c|}{ Bilbergia amoena (Loddiges) Lindley } \\
\hline \multicolumn{4}{|c|}{ Edmundoa lindenii (Regel) Leme } \\
\hline \multicolumn{4}{|l|}{ Nidularium innocentii Lemaire } \\
\hline \multicolumn{4}{|l|}{ Nidularium procerum Lindman } \\
\hline \multicolumn{4}{|l|}{ Wittrockia smithii Reitz } \\
\hline Wittrockia superba Lindman & & & \\
\hline
\end{tabular}

\section{Subfamília Tillandsioideae}

Racinaea spiculosa (Grisebach) M.A.Spencer \& L.B. Smith

Tillandsia gardneri Lindley

Tillandsia geminiflora Brongniart

Tillandsia stricta Solander

Tillandsia tenuifolia Linnaeus

Vriesea altodaserrae L. B. Smith

Vriesea cf atra Mez.

Vriesea carinata Wawra.

Vriesea erythrodactylon E. Morren ex Mez

Vriesea flammea L. B. Smith

Vriesea gigantea Gaudichaud

Vriesea incurvata Gaudichaud

Vriesea philippocoburgii Wawra

Vriesea platynema Gaudichaud

Vriesea rodigasiana $\mathrm{E}$. Morren

Vriesea vagans (L. B. Smith) L. B. Smith

Plântulas

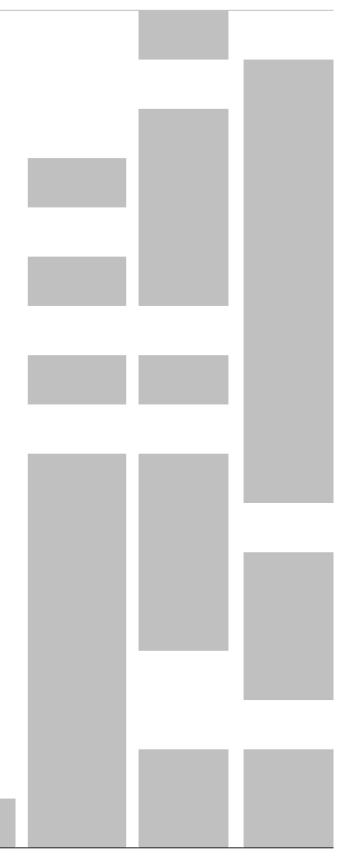

na região de domínio da Floresta Atlântica (Waechter 1992; Gatti 2000; Bonnet et al. 2007), e no território catarinense (Matos 2000; Rogalski 2002; Tiepo 2005; Bonnet \& Queiroz 2006). As diferenças encontradas na riqueza e composição das espécies entre as áreas amostradas evidenciam a sensibilidade das bromélias às mudanças e perturbações ambientais que ocorrem em cada estádio sucessional de regeneração da floresta amostrada e também o tempo em que os substratos estão disponíveis a colonização.

Dentre as características morfológicas dos forófitos, o diâmetro foi a variável que apresentou maior influência na riqueza das bromélias em todos os estádios com registro de bromélias epifíticas. A relação positiva registrada entre a riqueza de 
Tabela 2 - Alturas médias e máximas, diâmetro médio à altura do solo e do peito e número de espécies forofíticas amostradas nos diferentes estádios sucessionais da Floresta Ombrófila Densa do Parque Botânico do Morro Baú/ Ilhota - SC. $(\mathrm{n}=60)$. (DAS = diâmetro à altura do solo; DAP = diâmetro à altura do peito).

Table 2 - Dendometric measurements (heights and DBH) and quantification of the phorophytes sampled at different sucessional stages of the Atlantic Rainforest at the Botanical Park of Morro Baú (Ilhota, Santa Catarina State - Brazil). ( $\mathrm{n}=60)$. (DSL = diameter at soil level; $\mathrm{DBH}=$ diameter at breast height).

\begin{tabular}{lcccc}
\hline Estádio & $\begin{array}{c}\text { Altura média (m) } \\
\text { e desvio padrão }\end{array}$ & Altura máxima (m) & $\begin{array}{c}\text { DAS /DAP médio (cm) } \\
\text { e desvio padrão }\end{array}$ & $\begin{array}{c}\mathbf{N}^{\mathbf{o}} \text { espécies } \\
\text { forofíticas }\end{array}$ \\
\hline Capoeirinha & $3,13 \pm 0,89$ & 7,35 & $4 \pm 2,05$ & 15 \\
Capoeira & $7,83 \pm 3,15$ & 12,60 & $8,9 \pm 3,15$ & 9 \\
Capoeirão & $12,72 \pm 2,84$ & 18 & $17,38 \pm 5,71$ & 18 \\
Floresta Secundária & $14,07 \pm 3,87$ & 25 & $22,53 \pm 14,34$ & 32 \\
Floresta Primária & $17,82 \pm 3,37$ & 27 & $36,77 \pm 22,57$ & 36 \\
\hline
\end{tabular}

Tabela 3 - Frequência absoluta e relativa e padrão de casca das espécies forofíticas amostradas nos estádios sucessionais da Floresta Ombrófila Densa do Parque Botânico do Morro Baú/llhota-SC. ( $a i=n^{0}$ de pontos que a espécie ocorre; $\mathrm{FA}=$ frequência absoluta; $\mathrm{FR}=$ frequência relativa; $\mathrm{A}=$ áspera; $\mathrm{L}=$ lisa; $\mathrm{R}=$ rugosa; $\mathrm{F}=$ fissurada; $\mathrm{P}=$ persistente; $\mathrm{D}=$ descamante; $*$ = número de indivíduos da espécie com o padrão de casca correspondente)

Table 3 - Occurrence frequencies (absolute and relative) and bark assessment of the phorophytes sampled at different successional stages of the Atlantic Rainforest at the Botanical Park of Morro Baú (Ilhota, Santa Catarina State - Brazil). (ai = number of occurring spots; $\mathrm{FA}=$ Absolute frequency; $\mathrm{FR}=$ Relative frequency; $\mathrm{A}=\operatorname{rough} ; \mathrm{L}=$ smooth; $\mathrm{R}=\operatorname{rugged} ; \mathrm{F}=$ fissured; $\mathrm{P}=$ persistent; $\mathrm{D}=$ peeling; * = number of individuals with bark assessment)

\begin{tabular}{|c|c|c|c|c|c|}
\hline FAMÍLIAS & ESPÉCIES & ai & FA & $\mathbf{F R}$ & PADRÃO CASCA \\
\hline \multicolumn{6}{|l|}{ CAPOEIRINHA } \\
\hline \multirow[t]{4}{*}{ ASTERACEAE } & Baccharis dracunculifolia DC. & 8 & 53,33 & 19,05 & $\mathrm{~A} / \mathrm{P}^{(12)^{*}}$ \\
\hline & Baccharis cf semiserrata Aug. DC & 3 & 20,00 & 7,14 & $\mathrm{~A} / \mathrm{P}^{(5)}$ \\
\hline & Vernonia tweedieana Baker & 1 & 6,67 & 2,38 & $\mathrm{~A} / \mathrm{P}^{(1)}$ \\
\hline & Eupatorium intermedium DC. & 1 & 6,67 & 2,38 & $\mathrm{~A} / \mathrm{P}^{(1)}$ \\
\hline EUPHORBIACEAE & Hyeronima alchorneoides Fr. All. & 1 & 6,67 & 2,38 & $\mathrm{~A} / \mathrm{P}^{(1)}$ \\
\hline \multirow[t]{2}{*}{ FABACEAE } & Machaerium sp. & 1 & 6,67 & 2,38 & $\mathrm{~L} / \mathrm{P}^{(1)}$ \\
\hline & Platymiscium floribundum Vog.-Zuber & 1 & 6,67 & 2,38 & $\mathrm{~L} / \mathrm{P}^{(1)}$ \\
\hline \multirow[t]{5}{*}{ MELASTOMATACEAE } & Leandra dasytricha (A.Gray.) Cogn. & 1 & 6,67 & 2,38 & $\mathrm{~A} / \mathrm{P}^{(2)}$ \\
\hline & Miconia cinnamomifolia (DC.) Naud. & 1 & 6,67 & 2,38 & $\mathrm{~L} / \mathrm{P}^{(1)}$ \\
\hline & Ossaea confertiflora (DC.) Triana & 1 & 6,67 & 2,38 & $\mathrm{~A} / \mathrm{P}^{(1)}$ \\
\hline & Tibouchina pilosa Cogn. & 9 & 60,00 & 21,43 & $\mathrm{~L} / \mathrm{P}^{(16)}$ \\
\hline & Tibouchina urvilleana (DC) Cogn. & 7 & 46,67 & 16,67 & $\mathrm{~L} / \mathrm{P}^{(11)}$ \\
\hline MYRSINACEAE & Myrsine coriacea Nad. & 5 & 33,33 & 11,90 & $\mathrm{~L} / \mathrm{P}^{(5)}$ \\
\hline PIPERACEAE & Piper mosenii C. DC. & 1 & 6,67 & 2,38 & $\mathrm{~L} / \mathrm{P}^{(1)}$ \\
\hline SAPINDACEAE & Cupania vernalis Cambess. & 1 & 6,67 & 2,38 & $\mathrm{~L} / \mathrm{P}^{(1)}$ \\
\hline \multicolumn{6}{|l|}{ CAPOEIRA } \\
\hline CLORANTHACEAE & Hedyosmum brasiliense Mart. & 5 & 33,33 & 12,50 & $\mathrm{~A} / \mathrm{P}^{(5)^{*}}$ \\
\hline CLUSIACEAE & Clusia criuva Cambess & 10 & 66,67 & 25,00 & $\mathrm{~A} / \mathrm{P}^{(15)} \mathrm{L} / \mathrm{P}^{(2)}$ \\
\hline EUPHORBIACEAE & Hyeronima alchorneoides Fr. All. & 2 & 13,33 & 5,00 & $\mathrm{R} / \mathrm{P}^{(2)}$ \\
\hline FABACEAE & Albizia austrobrasilica A. Burkart & 2 & 13,33 & 5,00 & $\mathrm{~A} / \mathrm{P}^{(2)}$ \\
\hline
\end{tabular}




\begin{tabular}{|c|c|c|c|c|c|}
\hline FAMÍLIAS & ESPÉCIES & ai & FA & FR & PADRÃO CASCA \\
\hline FLACOURTICACEAE & Casearia decandra Jacq. & 1 & 6,67 & 2,50 & $\mathrm{~A} / \mathrm{P}^{(1)}$ \\
\hline MELASTOMATACEAE & Miconia cabucu W. Hoehne & 2 & 13,33 & 5,00 & $\mathrm{~F} / \mathrm{P}^{(2)}$ \\
\hline MYRSINACEAE & Myrsine coriacea Nad. & 14 & 93,33 & 35,00 & $\mathrm{~A} / \mathrm{P}^{(27)}$ \\
\hline \multirow[t]{2}{*}{ MYRTACEAE } & Myrcia rostrata DC. & 1 & 6,67 & 2,50 & $\mathrm{~A} / \mathrm{D}^{(1)}$ \\
\hline & Psidium guajava $\mathrm{L}$. & 3 & 20,00 & 7,50 & $\mathrm{~L} / \mathrm{D}^{(3)}$ \\
\hline \multicolumn{6}{|l|}{ CAPOEIRÃO } \\
\hline ASTERACEAE & Piptocarpha tomentosa Baker & 1 & 5,56 & 2,38 & $\mathrm{R} / \mathrm{D}^{(3)} \mathrm{F} / \mathrm{D}^{(1)^{*}}$ \\
\hline CECROPIACEAE & Cecropia glazioui Snethlage & 1 & 5,56 & 2,38 & $\mathrm{~L} / \mathrm{P}^{(1)}$ \\
\hline CYATHEACEAE & Cyathea schanschin Mart. & 1 & 5,56 & 2,38 & $\mathrm{~A} / \mathrm{P}^{(1)}$ \\
\hline EUPHORBIACEAE & Hyeronima alchorneoides Fr. All. & 8 & 44,44 & 19,05 & $\mathrm{R} / \mathrm{P}^{(9)} \mathrm{A} / \mathrm{P}^{(1)}$ \\
\hline FABACEAE & Platymiscium floribundum Vog.-Zuber & 1 & 5,56 & 2,38 & $\mathrm{~A} / \mathrm{P}^{(1)}$ \\
\hline \multirow[t]{2}{*}{ FLACOURTIACEAE } & Casearia decandra Jacq. & 1 & 5,56 & 2,38 & $\mathrm{~A} / \mathrm{P}^{(1)}$ \\
\hline & Casearia sylvestris $\mathrm{Sw}$. & 6 & 33,33 & 14,29 & $\mathrm{R} / \mathrm{P}^{(7)} \mathrm{A} / \mathrm{P}^{(1)}$ \\
\hline \multirow[t]{3}{*}{ LAURACEAE } & Endlicheria paniculata (Spreng.) Macbride & 1 & 5,56 & 2,38 & $\mathrm{~L} / \mathrm{P}^{(1)}$ \\
\hline & Nectandra membranacea Griseb. & 1 & 5,56 & 2,38 & $\mathrm{R} / \mathrm{P}^{(1)}$ \\
\hline & Nectandra sp. & 1 & 5,56 & 2,38 & $\mathrm{R} / \mathrm{P}^{(1)}$ \\
\hline MELASTOMATACEAE & Miconia cinnamomifolia Naud. & 11 & 61,11 & 26,19 & $\mathrm{~F} / \mathrm{P}^{(14)} \mathrm{R} / \mathrm{P}^{(4)}$ \\
\hline \multirow[t]{2}{*}{ MELIACEAE } & Cabralea canjerana (Vell.) Mart. & 1 & 5,56 & 2,38 & $\mathrm{~A} / \mathrm{P}^{(1)}$ \\
\hline & Cedrela fissilis Vell. & 2 & 11,11 & 4,76 & $\mathrm{~F} / \mathrm{P}^{(2)}$ \\
\hline MIRISTICACEAE & Virola bicuhyba Warb. & 1 & 5,56 & 2,38 & $\mathrm{~F} / \mathrm{P}^{(1)}$ \\
\hline MYRSINACEAE & Myrsine coriacea Nad. & 2 & 11,11 & 4,76 & $\mathrm{~A} / \mathrm{P}^{(4)}$ \\
\hline MYRTACEAE & Myrcia rostrata DC. & 1 & 5,56 & 2,38 & $\mathrm{R} / \mathrm{P}^{(2)}$ \\
\hline SAPINDACEAE & Matayba guianensis Aubl. & 1 & 5,56 & 2,38 & $\mathrm{R} / \mathrm{P}^{(1)}$ \\
\hline SOLANACEAE & Solanum pseudoquina A.St.Hil. & 1 & 5,56 & 2,38 & $\mathrm{R} / \mathrm{P}^{(1)}$ \\
\hline \multicolumn{6}{|c|}{ FLORESTA SECUNDÁRIA } \\
\hline ANACARDIACEAE & Tapirira guianensis Aubl. & 4 & 12,5 & 8 & $\mathrm{~F} / \mathrm{D}^{(1)} \mathrm{R} / \mathrm{D}^{(1)^{*}} \mathrm{R} / \mathrm{P}^{(2)}$ \\
\hline APOCYNACEAE & Aspidosperma olivaceum Muell Arg. & 1 & 3,13 & 2 & $\mathrm{~A} / \mathrm{P}^{(1)}$ \\
\hline ARECACEAE & Euterpe edulis Mart. & 4 & 12,5 & 8 & $\mathrm{~A} / \mathrm{P}^{(8)}$ \\
\hline \multirow[t]{2}{*}{ CYATHEACEAE } & Cyathea cf. phalerata Mart. & 1 & 3,13 & 2 & $\mathrm{~A} / \mathrm{D}^{(1)}$ \\
\hline & Alsophila setosa Kaulf.. & 1 & 3,13 & 2 & $\mathrm{R} / \mathrm{D}^{(1)}$ \\
\hline ELAEOCARPACEAE & Sloanea guianensis Benth. & 1 & 3,13 & 2 & $\mathrm{R} / \mathrm{P}^{(1)}$ \\
\hline \multirow[t]{3}{*}{ EUPHORBIACEAE } & Hyeronima alchorneoides Fr. All. & 1 & 3,13 & 2 & $\mathrm{R} / \mathrm{D}^{(1)}$ \\
\hline & Pausandra morisiana (Casar.) Radlk. & 2 & 6,25 & 4 & $\mathrm{~A} / \mathrm{P}^{(2)}$ \\
\hline & Pera glabrata (Schott) Baill. & 1 & 3,13 & 2 & $\mathrm{~A} / \mathrm{D}^{(1)}$ \\
\hline FABACEAE & Abarema langsdorffii Benth & 1 & 3,13 & 2 & $\mathrm{R} / \mathrm{P}^{(1)}$ \\
\hline HUMIRIACEAE & Vantanea compacta (Schnizl.) Cuatr. & 2 & 6,25 & 4 & $\mathrm{R} / \mathrm{P}^{(1)} \mathrm{R} / \mathrm{D}^{(1)}$ \\
\hline \multirow[t]{3}{*}{ LAURACEAE } & Aniba firmula (Nees) Mez. & 2 & 6,25 & 4 & $\mathrm{R} / \mathrm{P}^{(2)}$ \\
\hline & Ocotea acyphilla (Ness) Mez. & 1 & 3,13 & 2 & $\mathrm{~A} / \mathrm{P}^{(1)}$ \\
\hline & Ocotea catharinensis Mez. & 1 & 3,13 & 2 & $\mathrm{R} / \mathrm{D}^{(1)}$ \\
\hline \multirow[t]{2}{*}{ MELASTOMATACEAE } & Miconia cabucu W. Hoehne & 1 & 3,13 & 2 & $\mathrm{R} / \mathrm{P}^{(1)}$ \\
\hline & Miconia ef budlejoidesTriana & 1 & 3,13 & 2 & $\mathrm{R} / \mathrm{P}^{(1)}$ \\
\hline MELIACEAE & Trichilia cf lepidota Mart. & 1 & 3,13 & 2 & $\mathrm{~A} / \mathrm{P}^{(1)}$ \\
\hline MONIMIACEAE & Mollinedia cf schottiana Perkins. & 1 & 3,13 & 2 & $\mathrm{R} / \mathrm{P}^{(1)}$ \\
\hline MYRISTICACEAE & Virola bicuhyba Warb. & 4 & 12,5 & 8 & $\mathrm{R} / \mathrm{P}^{(4)} \mathrm{F} / \mathrm{P}^{(1)}$ \\
\hline MYRSINACEAE & Rapanea acuminata $\mathrm{Mez}$ & 1 & 3,13 & 2 & $\mathrm{R} / \mathrm{P}^{(1)}$ \\
\hline \multirow[t]{2}{*}{ MYRTACEAE } & Calyptranthes strigipes O. Berg. & 1 & 3,13 & 2 & $\mathrm{~A} / \mathrm{D}^{(1)} \mathrm{A} / \mathrm{P}^{(1)}$ \\
\hline & Eugenia cereja D. Legrand & 1 & 3,13 & 2 & $\mathrm{~A} / \mathrm{D}^{(1)}$ \\
\hline
\end{tabular}




\begin{tabular}{|c|c|c|c|c|c|}
\hline FAMÍLIAS & ESPÉCIES & ai & FA & FR & PADRÃO CASCA \\
\hline & Eugenia melanogyna (D. Legrand) Sobral & 1 & 3,13 & 2 & $\mathrm{~A} / \mathrm{D}^{(1)}$ \\
\hline & Eugenia obovata O. Berg. & 2 & 6,25 & 4 & $\mathrm{~A} / \mathrm{D}^{(1)} \mathrm{A} / \mathrm{P}^{(1)} \mathrm{R} / \mathrm{P}^{(1)}$ \\
\hline & Marlierea parviflora $\mathrm{O}$. Berg. & 1 & 3,13 & 2 & $\mathrm{R} / \mathrm{D}^{(1)}$ \\
\hline & Myrcia pubipetala Miq. & 1 & 3,13 & 2 & $\mathrm{~A} / \mathrm{D}^{(1)}$ \\
\hline & Myrcia spectabillis DC. & 1 & 3,13 & 2 & $\mathrm{~A} / \mathrm{D}^{(1)}$ \\
\hline NYCTAGINACEAE & Guapira opposita (Vell.) Reitz & 4 & 12,5 & 8 & $\mathrm{~L} / \mathrm{P}^{(1)} \mathrm{A} / \mathrm{P}^{(4)} \mathrm{R} / \mathrm{P}^{(1)}$ \\
\hline \multirow[t]{2}{*}{ RUBIACEAE } & Bathysa australis K. Schum. & 3 & 9,38 & 6 & $\mathrm{~A} / \mathrm{D}^{(4)}$ \\
\hline & Psychotria carthagenensis Jacq. & 1 & 3,13 & 2 & $\mathrm{R} / \mathrm{P}^{(1)}$ \\
\hline SABIACEAE & Meliosma sellowii Urb. & 1 & 3,13 & 2 & $\mathrm{R} / \mathrm{P}^{(1)}$ \\
\hline SAPOTACEAE & Pouteria cf venosa (Mart.) Baehni & 1 & 3,13 & 2 & $\mathrm{R} / \mathrm{P}^{(1)}$ \\
\hline \multicolumn{6}{|l|}{ FLORESTA PRIMÁRIA } \\
\hline COMBRETACEAE & Buchenavia kleinii M.Exell & 1 & 2,78 & 1,75 & $\mathrm{~A} / \mathrm{P}^{(1)}$ \\
\hline CHRYSOBALANACEAE & Hirtella hebeclata Moric. ex A.DC. & 2 & 5,56 & 3,51 & $\mathrm{~A} / \mathrm{P}^{(2)}$ \\
\hline ELAEOCARPACEAE & Sloanea guianensis Benth & 2 & 5,56 & 3,51 & $\mathrm{R} / \mathrm{P}^{(1)}, \mathrm{A} / \mathrm{P}^{(1)}$ \\
\hline EUPHORBIACEAE & Alchornea triplinervia Muell. Arg. & 3 & 8,33 & 5,26 & $\mathrm{R} / \mathrm{P}^{(1)}, \mathrm{R} / \mathrm{D}^{(1)}, \mathrm{F} / \mathrm{P}^{(1)}$ \\
\hline \multirow[t]{4}{*}{ FABACEAE } & Copaifera trapezifolia Hayne & 1 & 2,78 & 1,75 & $\mathrm{~A} / \mathrm{P}^{(1)}$ \\
\hline & Machaerium cf aculeatum Raddi & 1 & 2,78 & 1,75 & $\mathrm{R} / \mathrm{D}^{(1)}$ \\
\hline & Ormosia arborea (Harnu) & 1 & 2,78 & 1,75 & $\mathrm{~A} / \mathrm{D}^{(1)}$ \\
\hline & Platymiscium floribundum Vog.-Zuber & 2 & 5,56 & 3,51 & $\mathrm{~F} / \mathrm{P}^{(1)}, \mathrm{R} / \mathrm{P}^{(1)}$ \\
\hline FLACOURTIACEAE & Casearia decandra Jacq. & 1 & 2,78 & 1,75 & $\mathrm{~A} / \mathrm{P}^{(1)}$ \\
\hline HIPPOCRATEACEAE & cf Salacia elliptica G.Don & 1 & 2,78 & 1,75 & $\mathrm{R} / \mathrm{D}^{(1)}$ \\
\hline HUMIRIACEAE & Vantanea compacta (Schnitzl.) Cualr & 1 & 2,78 & 1,75 & $\mathrm{~F} / \mathrm{P}^{(1)}$ \\
\hline \multirow[t]{3}{*}{ LAURACEAE } & Cryptocaria aschersoniana $\mathrm{Mez}$ & 1 & 2,78 & 1,75 & $\mathrm{~A} / \mathrm{D}^{(1)}, \mathrm{R} / \mathrm{D}^{(1)}$ \\
\hline & Endicleria paniculata (Spreng) Macbride & 1 & 2,78 & 1,75 & $\mathrm{~A} / \mathrm{P}^{(2)}$ \\
\hline & Ocotea catharinensis $\mathrm{Mez}$ & 2 & 5,56 & 3,51 & $\mathrm{R} / \mathrm{P}^{(2)}$ \\
\hline LECYTHIDACEAE & Cariniana estrellensis (Raddi) Kuntze & 2 & 5,56 & 3,51 & $\mathrm{~F} / \mathrm{P}^{(1)}, \mathrm{R} / \mathrm{P}^{(1)}$ \\
\hline MALPHIGHIACEAE & Byrsonima ligustrifolia Jussieu & 2 & 5,56 & 3,51 & $\mathrm{R} / \mathrm{P}^{(2)}$ \\
\hline MELASTOMATACEAE & Mouriri chamisoana Cogn. & 2 & 5,56 & 3,51 & $\mathrm{~A} / \mathrm{D}^{(2)}$ \\
\hline MELIACEAE & Cedrela fissilis Vell. & 1 & 2,78 & 1,75 & $\mathrm{~F} / \mathrm{P}^{(1)}$ \\
\hline MONIMIACEAE & Mollinedia sp. & 1 & 2,78 & 1,75 & $\mathrm{R} / \mathrm{P}^{(1)}$ \\
\hline \multirow[t]{7}{*}{ MYRTACEAE } & Calyptranthes strigipes O.Berg & 2 & 5,56 & 3,51 & $\mathrm{~A} / \mathrm{D}^{(2)}$ \\
\hline & Eugenia handroana D. Legrand & 1 & 2,78 & 1,75 & $\mathrm{~A} / \mathrm{P}^{(1)}$ \\
\hline & Eugenia obovata O.Berg & 2 & 5,56 & 3,51 & $\mathrm{~A} / \mathrm{P}^{(2)}$ \\
\hline & Marlieria silvatica Kiaersk. & 1 & 2,78 & 1,75 & $\mathrm{~A} / \mathrm{P}^{(1)}$ \\
\hline & Marlierea tomentosa Cambess. & 3 & 8,33 & 5,26 & $\mathrm{R} / \mathrm{D}^{(1)}, \mathrm{A} / \mathrm{D}^{(1)}$ \\
\hline & Myrcia richardiana (O. Berg) Kiaersk. & 1 & 2,78 & 1,75 & $\mathrm{R} / \mathrm{P}^{(1)}$ \\
\hline & Myrcia rostrata DC. & 1 & 2,78 & 1,75 & $\mathrm{~A} / \mathrm{P}^{(1)}$ \\
\hline NYCTAGINACEAE & Guapira opposita(Vell.) Reitz & 2 & 5,56 & 3,51 & $\mathrm{~A} / \mathrm{P}^{(2)}$ \\
\hline OLACACEAE & Heisteria silvianii Schwacke & 7 & 19,4 & 12,3 & $\mathrm{R} / \mathrm{P}^{(4)}, \mathrm{A} / \mathrm{P}^{(2)}, \mathrm{R} / \mathrm{D}^{(2)}$ \\
\hline OLEACEAE & Chionanthus trichotomus (Vell.) P.S. Green & 1 & 2,78 & 1,75 & $\mathrm{R} / \mathrm{P}^{(1)}$ \\
\hline \multirow[t]{3}{*}{ RUBIACEAE } & Bathysa australis K.Schum. & 1 & 2,78 & 1,75 & $\mathrm{R} / \mathrm{P}^{(1)}$ \\
\hline & Chomelia sp. & 1 & 2,78 & 1,75 & $\mathrm{~A} / \mathrm{P}^{(1)}$ \\
\hline & Posoqueria latifolia Roem. \& Schult. & 1 & 2,78 & 1,75 & $\mathrm{~A} / \mathrm{P}^{(1)}$ \\
\hline SAPINDACEAE & Cupania vernalis Cambess. & 2 & 5,56 & 3,51 & $\mathrm{R} / \mathrm{P}^{(2)}$ \\
\hline \multirow[t]{2}{*}{ SAPOTACEAE } & Crysophyllum dusenii Cronquist & 1 & 2,78 & 1,75 & $\mathrm{R} / \mathrm{P}^{(1)}$ \\
\hline & Pouteria venosa (Mart.) Baehni & 1 & 2,78 & 1,75 & $\mathrm{~A} / \mathrm{D}^{(1)}$ \\
\hline VERBENACEAE & Lamanonia speciosa (Cambess.) L.B.Smith & 1 & 2,78 & 1,75 & $\mathrm{R} / \mathrm{P}^{(1)}$ \\
\hline
\end{tabular}


Tabela 4 - Relação entre o número de espécies de bromélias epifíticas, o DAP (diâmetro à altura do peito $=1,30 \mathrm{~m}$ ) e altura, obtida pela Correlação de Spearman, nos diferentes estádios sucessionais da Floresta Ombrófila Densa, Parque Botânico do Morro Baú - Ilhota/SC. (*significativo $\mathrm{p}<0,05 ; \mathrm{n}=60$ ).

Table 4 - Relationship between the number of epiphytic bromeliad species with height and DBH obtained by Spearman Correlation at different successional stages of the Atlantic Rainforest at the Botanical Park of Morro Baú (Ilhota, Santa Catarina State - Brazil). (*significant $\mathrm{p}<0,05 ; \mathrm{n}=60)$.

\begin{tabular}{lcc}
\hline Estádios & DAP & Altura \\
\hline Capoeirão & Correlação & Correlação \\
Floresta Secundária & $0,32^{*}$ & $0,20^{*}$ \\
Floresta Primária & $0,60^{*}$ & $0,56^{*}$ \\
\hline
\end{tabular}

bromélias, o diâmetro e altura dos forófitos indica uma possível influência do tempo em que os forófitos se encontram disponíveis à colonização, a disponibilidade de espaço colonizável e com os gradientes microclimáticos que podem ser oferecidos ao longo de todo o forófito (Benzing 1995a). O aumento desta correlação ocorre progressivamente em direção à Floresta Primária, local onde há maior expressividade dos forófitos, tanto em diâmetro quanto em altura, e também por apresentar um maior número de árvores mais antigas em comparação a outros estádios. De igual modo, resultados positivos entre riqueza e DAP também foram encontrados por Dias (2009), onde demonstrou que ambientes com maior quantidade de árvores possuem mais locais de estabelecimento para epífitas, e que ambientes com árvores de grande porte estão a mais tempo suscetíveis ao estabelecimento de propágulos de espécies epifíticas. Consequentemente, estes locais tendem a ter maior abundância e riqueza de epífitas que ambientes com regeneração recente. Igualmente

Tabela 5 - Padrão de casca com maior freqüência de bromélias e forófitos mais frequentes por estádio sucessional e por estágio de desenvolvimento das bromélias epífitas em ambientes de Floresta Ombrófila Densa no Parque Botânico do Morro Baú/Ilhota - SC.

Table 5 - Bark assessment with a higher bromeliads frequency and phorophytes frequently by sucessional stage and stage epiphytic bromeliads development in Atlantic Rainforest environments at the Botanical Park of Morro do Baú (Ilhota, Santa Catarina State - Brazil).

\begin{tabular}{|c|c|c|c|}
\hline Estádio Sucessional & $\begin{array}{l}\text { Estágio } \\
\text { Bromélia }\end{array}$ & Padrão de Casca com maior frequencia de bromélias & $\begin{array}{l}\text { Forófitos mais frequentes/ Tipo } \\
\text { de Casca }\end{array}$ \\
\hline & Adulta & Rugosa e Fissurada & Miconia cinnamomifolia - fissurada \\
\hline & & $\left(X^{2}\right.$ rugosa $x$ fissurada $\left.=1,16 ; G L=1 ; p>0,05\right)$ & Hyeronima alchorneoides - rugosa \\
\hline \multirow[t]{6}{*}{ Capoeirão } & Plântula & Rugosa e Fissurada & Casearia silvestris - rugosa \\
\hline & & $\left(X^{2}\right.$ rugosa $\times$ fissurada $\left.=0,05 ; G L=1 ; p>0,05\right)$ & \\
\hline & & Persistente & \\
\hline & & $\left(X^{2}\right.$ persistente $x$ descamante $\left.=28 ; 3 G L=1 ; p<0,05\right)$ & \\
\hline & Adulta & Áspera e Rugosa & Euterpe edulis - áspera \\
\hline & & $\left(X^{2}\right.$ áspera $\times$ rugosa $\left.=0,58 ; G L=1 ; p>0,05\right)$ & Guapira opposita - áspera \\
\hline \multirow[t]{5}{*}{ Floresta Secundária } & Plântula & Áspera e Rugosa & Virola bicuhyba - áspera \\
\hline & & $\left(X^{2}\right.$ áspera $\times$ rugosa $\left.=0,32 ; G L=1 ; p>0,05\right)$ & \\
\hline & & Persistente & \\
\hline & & $\left(X^{2}\right.$ persistente $x$ descamante $\left.=5,0 ; G L=1 ; p<0,05\right)$ & \\
\hline & Adulta & Áspera e Rugosa & Heisteria silvianii - rugosa \\
\hline \multirow[t]{5}{*}{ Floresta Primária } & & $\left(X^{2}\right.$ áspera $\times$ rugosa $\left.=0,30 ; G L=1 ; p>0,05\right)$ & \\
\hline & Plântula & Áspera e Rugosa & \\
\hline & & $\left(X^{2}\right.$ áspera $\times$ rugosa $\left.=0,30 ; G L=1 ; p>0,05\right)$ & \\
\hline & & Persistente & \\
\hline & & $\left(X^{2}\right.$ persistente $x$ descamante $\left.=30 ; G L=1 ; p<0,05\right)$ & \\
\hline
\end{tabular}


Bonnet et al. (2007) destacam as dimensões e o tempo de exposição dos forófito como elementos fundamentais na definição da riqueza de bromélias epifíticas nos estádios mais avançados da sucessão. Flores-Palacios \& Garcia-Franco (2006) também afirmam existir relação positiva e linear entre o tamanho dos forófitos e a riqueza de epífitas que suportam.

A preferência da ocorrência de bromélias em forófitos com cascas ásperas e rugosas indica que espécies arbóreas de casca lisa são as menos adequadas à colonização por epífitas. Em geral, quanto maior a rugosidade presente na casca das árvores, maior a capacidade de retenção de umidade e também de matéria orgânica (Ter Steege \& Cornelissen 1989; Benzing 1990). Já a estabilidade do substrato pode interferir não só no processo de estabelecimento dos diásporos, mas também sobre plântulas já estabelecidas, dependendo, é claro, do grau de descamação da espécie e do local de fixação da bromélia. Segundo Kersten (2010), a capacidade de retenção de umidade pela casca influencia o estabelecimento de plântulas, para os quais pequenas quantidades de água são suficientes. Já a morfologia da casca influencia o estabelecimento dos diásporos, a umidade e a quantidade de nutrientes (Benzing 1995b).

Desse modo, em trabalhos de recuperação de áreas degradadas, o uso de espécies com casca persistente e rugosa/fissurada pode facilitar a fixação e o desenvolvimento de bromélias epifíticas na área, favorecendo o aumento da biodiversidade $\mathrm{e}$ umidade local, e consequentemente o aparecimento de outras espécies da fauna e da flora.

\section{Agradecimentos}

Agradecemos à CAPES, a concessão de bolsa à primeira autora deste artigo; ao biólogo Marcelo Kammers, as sugestões feitas ao trabalho; ao Arnaldo Ferreira e à Heloisa Dallanhol, a tradução do abstract e revisão do manuscrito; à equipe Helarita, Ingo, Michelle, Silvanio e Fernando, o apoio nas saídas de campo e aos revisores pelas sugestões.

\section{Referências}

Almeida, D.R.; Carvalho, L.C. \& Rocha, C.F.D. 1998. As bromeliáceas da Mata Atlântica da Ilha Grande, RJ: composição e diversidade de espécies em três ambientes diferentes. Bromélia 5: 55-65.

Akinsoji, A. 1990. Studies on epiphytic flora of a tropical rain forest in southwestern Nigeria. Vegetatio 88: 87-92.
Bennet, B.C. 1986. Patchiness, diversity, and abundance relationships of vascular epiphytes. Selbyana 9: 70-75.

Benzing, D.H. 1981. Bark surfaces and the origin and maintenance of diversity among angiosperm epiphytes: a hypothesis. Selbyana 5: 258-255.

Benzing, D.H. 1995a. Vascular epiphytes. In: Lowman, M.D. \& Nadkarni, N.M. (ed.). 1995. Forest Canopies. Academic Press, San Diego. 544p.

Benzing, D.H. 1995b. The physical mosaic and plant variety in forest canopies. Selbyana 16: 159-168.

Benzing, D.H. 2000. Bromeliaceae: profile of an adaptative radiation. Cambridge University Press, Cambridge. 690p.

Benzing, D.H. \& Renfrow, A. 1974. The mineral nutrition of Bromeliaceae. Botanical Gazete 135: 281-288.

Bonnet, A.; \& Queiroz, M.H. 2006. Estratificação vertical de bromélias epifíticas em diferentes estádios sucessionais da floresta ombrófila densa, Ilha de Santa Catarina, Santa Catarina, Brasil. Revista Brasileira de Botânica 29: 217-228.

Bonnet, A.; Queiroz, M.H.; Lavoranti, O.J. 2007. Relações de bromélias epifíticas com características dos forófitos em diferentes estádios sucessionais da Floresta Ombrófila Densa, Santa Catarina, Brasil. Floresta 37: 83-94.

Borgo, M. \& Silva, S.M. 2003. Epífitos vasculares em fragmentos de Floresta Ombrófila Mista, Curitiba, Paraná, Brasil. Revista Brasileira de Botânica 26: 391-401.

Braun-Blanquet, J. 1979. Bases para el estudio de las comunidades vegetales. Blume Ediciones, Rosário. $820 \mathrm{p}$.

Breier, T.B. 1999. Florística e ecologia de epífitos vasculares em uma Floresta Costeira do Sul do Brasil. Dissertação de Mestrado. Universidade Federal do Rio Grande do Sul, Porto Alegre. 83 p.

Budowski, G. 1965. Distribution of tropical american rain forest species in the light of succesional processes. Turrialba 15: 40-42.

Callaway, R.M.; Reinhart, K.O.; Moore, G.W.; Moore, D.J. \& Pennings, S.C. 2002. Epiphyte host preferences and host traits: mechanisms for speciesspecific interactions. Oecologia 132: 221-230.

Carlsen, M. 2000. Structure and diversity of the vascular epiphyte community in the overstory of a tropical rain forest in Surumoni, Amazonas State, Venezuela. Selbyana 21: 7-10.

Cogliatti-Carvalho, L.; Rocha, C.F.D.; Freitas, A.F.N. \& Rocha-Pessôa, T.C. 2001. As bromélias da Ilha Grande. Bromélia 6: 7-11.

Dias, A.S. 2009. Ecologia de epífitas vasculares em uma área de Mata Atlântica do Parque Nacional da Serra dos Órgãos, Teresópolis, RJ. 2009. Dissertação de Mestrado. Universidade Federal Rural do Rio de Janeiro, Rio de Janeiro. 61p.

Dejean, A.; Olmstead, I. \& Snelling, R.R. 1995. Treeepiphyte-ant relationships in the low inundated 
forest of the Sian Ka'an Biosphere Reserve, Quintana Roo, Mexico. Biotropica 27: 57-70.

Fischer, E. \& Araujo, A.C. 1995. Spatial organization of a bromeliad community in the Atlantic rainforest, south-eastern Brazil. Journal of Tropical Ecology 11: 559-567.

Flores-Palacios, A. \& García-Franco, G. 2006. The relationship between tree size and epiphyte species richness: testing four different hypotheses. Journal of Biogeography 33: 323-330.

Fontoura, T.; Sylvestre, L.S.; Vaz, A.M.S; \& Vieira, C.M. 1997. Epífitas vasculares, hemiepífitas e hemiparasitas da Reserva Ecológica de Macaé de Cima. In: Lima, H.C. \& Guedes-Bruni, R.R. (ed.) Serra de Macaé de Cima: diversidade florística e conservação da Mata Atlântica. Editora do Jardim Botânico, Rio de Janeiro. Pp. 89-101.

Fontoura, T.; Rocca, M.A.; Schilling, A.C. \& Reinert, F. 2009. Epífitas da floresta seca da Reserva Ecológica Estadual de Jacarepiá, sudeste do Brasil: relações com a comunidade arbórea. Rodriguésia 60: 171-185.

Frei, J.K. \& Dodson, C.H. 1972. The chemical effect of certain bark substrates on the germination of early growth epiphytic orchids. Bulletin of the Torrey Botanical Club 99: 301-307.

Gaplan. 1986. Gabinete de Planejamento e Coordenação Geral. Atlas de Santa Catarina. Rio de Janeiro: Aerofoto Cruzeiro. 173p.

Gatti, A.L.S. 2000. O componente epifítico vascular na Reserva Natural de Salto Morato, Guaraqueçaba PR. Dissertação de Mestrado. Universidade Federal do Paraná, Paraná. 93p.

Gentry, A.H \& Dodson, C.H. 1987. Diversity and biogeography of neotropical vascular epiphytes. Annals of the Missouri Garden 74: 205-233.

Hietz, P. 1997. Population dynamics of epiphytes in a Mexican humid montane forest. Journal of Ecology 85: 767-775.

Hietz, P. \& Hietz-Seifert, U. 1995. Intra and interspecific relations within an epiphyte community in a Mexican Humid Montane Forest. Selbyana 16: 135-140.

Hietz-Seifert, U.; Hietz, P. \& Guevara, S. 1996. Epiphyte vegetation and diversity on remnant trees after forest clearance in southern Veracruz, Mexico. Biological Conservation 75: 103-111.

Hirata, A.; Kamijo, T. \& Saito, S. 2009. Host trait preferences and distribution of vascular epiphytes in a warm-temperate forest. Plant Ecology 201: 247-254.

Kernan, C. \& Fowler, N. 1995. Differential substrate use by epiphytes in Corcovado National Park, Costa Rica: a source of guild structure. Journal Ecology 83: 65-73.

Kersten, R.A. \& Silva, S.M. 2001. Composição florística do componente epifítico vascular em floresta da planície litorânea na Ilha do Mel, Paraná, Brasil. Revista Brasileira de Botânica 24: 213-226.
Kersten, R.A.; Borgo, M. \& Silva, S.M. 2009. Diversity and distribution of vascular epiphytes in an insular Brazilian coastal forest. International Journal of Tropical Biology 57: 749-759.

Kersten, R.A. 2010. Epífitas vasculares - histórico, participação taxonômica e aspectos relevantes, com ênfase na Mata Atlântica. Hoehnea 37: 9-38.

Klein, R.M. 1980. Ecologia da flora e vegetação do Vale do Itajaí. Sellowia 31-32: 9-389.

Krebs, C.J. 1999. Ecological methodology. 2nd ed. Benjamin Cummings, California. 620p.

Leme, E.M. \& Marigo, L.C. 1993. Bromélias na natureza. Marigo Comunicação Visual, Rio de Janeiro. 183p.

Martins, F.R. 1993. Estrutura de uma floresta mesófila. $2^{\mathrm{a}}$ ed. Ed.Unicamp, Campinas. 246p.

Matos, J.Z. 2000. Ecologia de bromélias com ênfase em Vriesea incurvata Gaud. (Bromeliaceae), em áreas com vegetação primária e secundária da floresta tropical atlântica, no sul do Brasil. Dissertação de Mestrado. Universidade Federal de Santa Catarina, Santa Catarina. 91p.

Medeiros, T.D.S. \& Jardim, M. 2011. Distribuição vertical de orquídeas epífitas na Área de Proteção Ambiental (APA) Ilha do Combu, Belém, Pará, Brasil. Revista Brasileira de Biociências 9: 33-38.

Migenis, L.E. \& Ackerman, J.D. 1993. Orchidsporophyte relationships in a forest watershed in Puerto Rico. Journal Tropical Ecology 9: 231-240.

Mueller-Dombois, D. \& Ellenberg, H. 1974. Aims and methods of vegetation ecology. John Wiley \& Sons, USA. 547 p.

Nieder, J.; Engwald, S.; Klawun, M. \& Barthlott, W. 2000. Spatial distribution of vascular epiphytes (including hemiepiphytes) in a lowland Amazonian rain Forest (Surumoni Crane Plot) of Southern Venezuela. Biotropica 32: 385-396.

Pittendrigh, C. S. 1948. The bromeliad-anophelesmalaria complex in Trinidad I. The bromeliad flora. Evolution 2: 58-89.

Queiroz, M.H. 1994. Approche phytoécologique et dynamique des formations vegetales secondaires développées aprés abandon des activités agricoles, dans le domaine de la forêt ombrophile dense (Forêt Atlantique) à Santa Catarina - Brésil. Tese de Doutorado. École Nationale du Génie Rural, des Eaux et des Forêts, Nancy, França. 250p.

Rogalski, J.M. 2002. Distribuição espacial de bromélias e aráceas epifíticas em diferentes situações topográficas de Floresta Ombrófila Densa, Ilha de Santa Catarina/ SC. Dissertação de Mestrado. Universidade Federal de Santa Catarina, Santa Catarina. 126p.

Schlesinger, W.H. \& Marks, P.L. 1977. Mineral cycling and the niche of spanish moss, Tillandsia usneoides L. American Botanical 64: 1254-1262.

Silva, J.C. 1994. Bromélias do Rio Grande do Sul - I. O Parque Estadual de Itapuã. Bromélia 1: 19-23. 
Sokal, R.R. \& Rohlf, F.J. 1969. Biometry. Freeman and Company, San Francisco. 937p.

Ter Steege, H. \& Cornelissen, J.H.C. 1989. Distribution and ecology of vascular epiphytes in lowland rain forest of Guyana. Biotropica 21: 331-339.

Tieppo, E.N. 2005. Bromélias em três estágios sucessionais na Unidade de Conservação Ambiental Desterro, Florianópolis/SC. Dissertação de Mestrado. Universidade Federal de Santa Catarina, Florianópolis. 69p.

Torres, R.B.; Kinoshita, L.S. \& Martins, F.R. 1994. Aplicação de padrões de casca na identificação de árvores da Estação Ecológica de Angatuba, SP. Revista Brasileira de Botânica 17: 119-127.

Waechter, J.L. 1992. O epifitismo vascular na planície costeira do Rio Grande do Sul. Tese de Doutorado. Universidade Federal de São Carlos, São Paulo. 163p.

Waechter, J.L. 1998. Epifitismo vascular em uma floresta de restinga do Brasil Subtropical. Revista Ciência e Natura 20: 43-66.

Zimmerman, J.K. \& Olmsted, I.C.1992. Host tree utilization by vascular epiphytes in a seasonally inundated forest (Tintal) Mexico. Biotropica 24: 402-407. 